\title{
Comparative study of the effects of different radiation qualities on normal human breast cells
}

Dajana Juerß ${ }^{1 *+}$, Monique Zwar ${ }^{1 \dagger}$, Ulrich Giesen², Ralf Nolte ${ }^{2}$, Stephan Kriesen ${ }^{1}$, Giorgio Baiocco ${ }^{3}$, Monika Puchalska ${ }^{4}$, Marc-Jan van Goethem ${ }^{5}$, Katrin Manda ${ }^{1}$ and Guido Hildebrandt ${ }^{1}$

\begin{abstract}
Background: As there is a growing number of long-term cancer survivors, the incidence of carcinogenesis as a late effect of radiotherapy is getting more and more into the focus. The risk for the development of secondary malignant neoplasms might be significantly increased due to exposure of healthy tissue outside of the target field to secondary neutrons, in particular in proton therapy. Thus far, the radiobiological effects of these neutrons and a comparison with photons on normal breast cells have not been sufficiently characterised.

Methods: MCF10A cells were irradiated with doses of up to 2 Gy with neutrons of different energy spectra and $X$-rays for comparison. The biological effects of neutrons with a broad energy distribution $\left(\left\langle E_{n}\right\rangle=5.8 \mathrm{MeV}\right)$, monoenergetic neutrons (1.2 MeV, $0.56 \mathrm{MeV})$ and of the mixed field of gamma's and secondary neutrons $\left(<E_{n}>=\right.$ $70.5 \mathrm{MeV}$ ) produced by $190 \mathrm{MeV}$ protons impinging on a water phantom, were analysed. The clonogenic survival and the DNA repair capacity were determined and values of relative biological effectiveness were compared. Furthermore, the influence of radiation on the sphere formation was observed to examine the radiation response of the potential fraction of stem like cells within the MCF10A cell population.

Results: X-rays and neutrons caused dose-dependent decreases of survival fractions after irradiations with up to $2 \mathrm{~Gy}$. Monoenergetic neutrons with an energy of $0.56 \mathrm{MeV}$ had a higher effectiveness on the survival fraction with respect to neutrons with higher energies and to the mixed gamma - secondary neutron field induced by proton interactions in water. Similar effects were observed for the DNA repair capacity after exposure to ionising radiation (IR). Both experimental endpoints provided comparable values of the relative biological effectiveness. Significant changes in the sphere formation were notable following the various radiation qualities.

Conclusion: The present study compared the radiation response of MCF10A cells after IR with neutrons and photons. For the first time it was shown that monoenergetic neutrons with energies around $1 \mathrm{MeV}$ have stronger radiobiological effects on normal human breast cells with respect to $X$ rays, to neutrons with a broad energy distribution $\left(<E_{n}\right\rangle=5$. $8 \mathrm{MeV}$, and to the mixed gamma - secondary neutron field given by interactions of $190 \mathrm{MeV}$ protons in water. The results of the present study are highly relevant for further investigations of radiation-induced carcinogenesis and are very important in perspective for a better risk assessment after secondary neutron exposure in the field of conventional and proton radiotherapy.
\end{abstract}

Keywords: Neutron irradiation, X-rays, Normal human breast cells, Relative biological effectiveness

\footnotetext{
* Correspondence: dajana.buttler@uni-rostock.de

${ }^{\dagger}$ Equal contributors

'Department of Radiotherapy and Radiation Oncology, University Medical

Centre Rostock, Suedring 75, 18059 Rostock, Germany

Full list of author information is available at the end of the article
} International License (http://creativecommons.org/licenses/by/4.0/), which permits unrestricted use, distribution, and reproduction in any medium, provided you give appropriate credit to the original author(s) and the source, provide a link to the Creative Commons license, and indicate if changes were made. The Creative Commons Public Domain Dedication waiver (http://creativecommons.org/publicdomain/zero/1.0/) applies to the data made available in this article, unless otherwise stated. 


\section{Background}

The incidence of carcinogenesis as a late effect of radiotherapy is discussed in several studies as there is a growing number of long-term cancer survivors [1-5]. The risk for the development of secondary malignant neoplasms is significantly increased, especially for breast cancers among women who were irradiated as a treatment for Hodgkin disease in childhood or adolescence using particle proton therapy [6-9], which allows a better dose conformation to the target volume than conventional radiotherapy. Due to a production of secondary neutrons during proton therapy (and also during photon therapy with high energies), healthy tissue distal to the target region can be exposed to neutrons with a potentially high biological effectiveness [10]. Until now, the potential for the induction of neoplasms following low-dose neutron exposures has not been very well characterised.

The DNA can be considered as the most important target of ionising radiation (IR), since misrepair of radiation-induced DNA damage can be the initial step of carcinogenesis. Hence, radiation-induced DNA doublestrand breaks (DSBs) were scored $24 \mathrm{~h}$ after exposure by visualising and counting residual $\gamma \mathrm{H} 2 \mathrm{AX}$ foci, which are considered as a reliable biomarker for the investigation of complex DNA damage [11-13]. The clonogenic survival was determined after neutron and X-ray IR via colony forming assays as a measure of long-term effects and to classify the unknown biological effects of a clinical relevant secondary neutron field into a defined spectrum of different neutron energies. Using X-rays as a reference, the observed radiation effects of neutrons were compared using the concept of relative biological effectiveness (RBE). Irradiations with low-energy monoenergetic neutrons and medium-energy neutrons with a broad energy distribution and a mean energy of about $5.8 \mathrm{MeV}$ were performed. In order to generate a field of secondary neutrons, similar to that produced during proton therapy, a $190 \mathrm{MeV}$ proton beam was directed onto a water phantom.

In 2009, Stingl suggested that "normal stem and progenitor cells are the likely targets for malignant transformation" and have the ability to self-renew [14]. In order to confirm the capacity of healthy mammary cells to function as progenitor cells, and thus as an initial target for carcinogenesis, the present study investigated the self-renewal potential of the MCF10A cells - a nontransformed cell line with properties of progenitor cells [15] - utilising a 3D spheres formation assay as described in literature $[14,16]$. Our study of these highly relevant endpoints and measurements of RBE values for neutron exposures will expand on the current knowledge: provided results are useful in perspective for the assessment of the risk of cancer induction by IR, both for radiation protection and for optimization in proton and photon therapy, through the inclusion in treatment planning of the risk evaluation for secondary cancer.

\section{Methods \\ Cell culture}

MCF10A (provided by Prof. Kevin Prise, Queen's University Belfast, Ireland), is a spontaneously transformed cell line from normal human mammary epithelial cells [17], authenticated using STR typing (Additional file 1). The cells were cultivated using Dulbecco's modified Eagle medium/F12 (DMEM/F12, Gibco/Life Technologies, Darmstadt, Germany) supplemented with $0.01 \%$ cholera toxin, $0.1 \%$ insulin, $0.05 \%$ hydrocortisone and 1\% penicillin/streptomycin (all Sigma Aldrich, Hamburg, Germany), 0.02\% epidermal growth factor (EGF; Gibco/ Life Technologies) and 5\% horse serum (Fisher Scientific, Schwerte, Germany) under $5 \% \mathrm{CO}_{2}$ and at $37{ }^{\circ} \mathrm{C}$. The cells were passaged two times a week.

\section{Irradiation setup}

A homogenous irradiation for cells in a single cell suspension was ensured by using rotating systems. The first setup consists in a ring holder with seven cylindrical containers (1 $\mathrm{ml}$ volume, $3 \mathrm{~mm}$ thick, $20 \mathrm{~mm}$ in diameter), made of polymethylmethacrylate (PMMA), positioned on a wooden motor-driven rotator, which allowed a slow rotation to keep the cells in suspension during the whole irradiation time. This arrangement was used for the irradiations with X-rays, medium-energy neutrons with a broad energy distribution at Physikalisch-Technische Bundesanstalt (PTB, Braunschweig, Germany) and for the mixed gamma - secondary neutron field at the KVI-Center for Advanced Radiation Technology (KVI-CART, Groningen, The Netherlands), where the radiation field was wide enough to perform homogeneous irradiation of more containers at a time with a single dose. For irradiations with low-energy monoenergetic neutrons at PTB the same containers but a modified rotator was used, on which three samples were placed in a row at distances of 50, 70 and $100 \mathrm{~mm}$ from the neutron source. This solution allowed to perform irradiation of three containers at a time, each distance corresponding to a different dose point.

Sham irradiated samples were used as negative control. Afterwards the cells were seeded as an adherent culture.

As the reference, photon irradiations with X-rays were performed at ambient temperature using an X-Strahl 200 system (Xstrahl Ltd., Surrey, United Kingdom) at $220 \mathrm{kV}$, filtered with $1 \mathrm{~mm} \mathrm{Al}, 0.25 \mathrm{~mm} \mathrm{Cu}$ and $0.45 \mathrm{~mm} \mathrm{Sn}$. The dosimetry is based on the German Standard DIN 6809-5. The uncertainties are about $\pm 3 \%$. In addition to irradiations at a high dose rate (HDR) of $0.37 \mathrm{~Gy} / \mathrm{min}$, a low dose rate (LDR) of $0.02 \mathrm{~Gy} / \mathrm{min}$ was 
used to match the dose rate of the neutron irradiations. Doses of 0.1, 0.25, 0.5, 1, 2, 4 and 6 Gy were used.

Two types of neutron irradiations were performed at PTB: firstly, a "medium-energy" intense neutron field with dose rates of $0.1 \mathrm{~Gy} / \mathrm{min}$ (HDR) and of about $0.003 \mathrm{~Gy} / \mathrm{min}$ (LDR). It was produced by the ${ }^{9} \mathrm{Be}+\mathrm{d}$ reaction on a thick Be-target within a collimator at a deuteron energy of $13 \mathrm{MeV}$. The energy distribution is broad and extends from about 0.1 to $10 \mathrm{MeV}$ [18]. The energy spectrum is shown in Fig. 1. The 0-degree, "freein-air", tissue-kerma-averaged mean neutron energy is $<E_{\mathrm{n}}>=5.8 \mathrm{MeV}[19]$ (for ionising radiation, kerma is defined as the sum of the initial kinetic energies of all charged particles liberated in a given mass of material by the incident uncharged particles, divided by such mass; the unit of kerma is Gy [20]). Dose to tissue was determined using a calibrated tissue-equivalent ionisation chamber according to ICRU89 [21]. The relative standard uncertainty for the total dose determination was $6 \%$. The dose due to photon radiation was about $2.5 \%$. Neutron doses of 0.1, 0.25, 0.5, 1 and 2 Gy were applied.

Secondly, "low-energy" monoenergetic neutrons with an energy of $1.2 \mathrm{MeV}(0.003 \mathrm{~Gy} / \mathrm{min})$ were produced by the $\mathrm{T}(\mathrm{p}, \mathrm{n})^{3} \mathrm{He}$ reaction and neutrons of $0.56 \mathrm{MeV}$ $(0.0045 \mathrm{~Gy} / \mathrm{min})$ by the ${ }^{7} \mathrm{Li}(\mathrm{p}, \mathrm{n})^{7} \mathrm{Be}$ reaction $[22,23]$. Both energy spectra are shown in Fig. 1. The neutron yield per unit target charge at an emission angle of $0^{\circ}$ was measured using a Long Counter. Monte Carlo neutron transport calculations were carried out to determine the spectral neutron distribution in the liquid cell suspensions from the $0^{\circ}$ neutron yield and the known angular distribution of neutron yield and neutron energy at larger emission angles. The tissue kerma was calculated from the spectral fluence using the fluence-tokerma conversion coefficients [19]. In this way, the contribution of neutrons scattered in the sample holder was properly accounted for. The relative contributions of scattered neutrons to the kerma and the kermaweighted mean energy of the scattered neutrons are summarised in Table 1 . The relative standard uncertainty for the total dose determination was about $7 \%$. The dominant contribution resulted from the uncertainty of the distance of the sample to the neutron source. Cells were exposed to neutrons of $0.56 \mathrm{MeV}$ with doses of $0.16 \mathrm{~Gy}, 0.40 \mathrm{~Gy}$ and $0.84 \mathrm{~Gy}$, and to neutrons of $1.2 \mathrm{MeV}$ with doses of $0.18,0.42$ and $0.85 \mathrm{~Gy}$ for colony forming and $\gamma \mathrm{H} 2 \mathrm{AX}$ assay, as well as doses of $0.82 \mathrm{~Gy}$ (for $0.56 \mathrm{MeV}$ ) and $0.88 \mathrm{~Gy}$ (for $1.2 \mathrm{MeV}$ ) for spheres formation assay.

In order to generate a neutron spectrum similar to that produced during proton therapy, additional irradiations were performed at the KVI-CART. An uncollimated pencil beam of $190 \mathrm{MeV}$ protons with a width $(1 \sigma)$ of $4 \mathrm{~mm}$ and an RMS energy spread of about $0.2 \%$ was directed onto a $300 \mathrm{~mm}$ cubic water phantom (with front and back layers of $8 \mathrm{~mm}$ PMMA) in which the protons were stopped. The beam profile at the entrance of the phantom was measured with Gafchromic EBT film. The proton current impinging on the water phantom was monitored using an ionisation chamber which
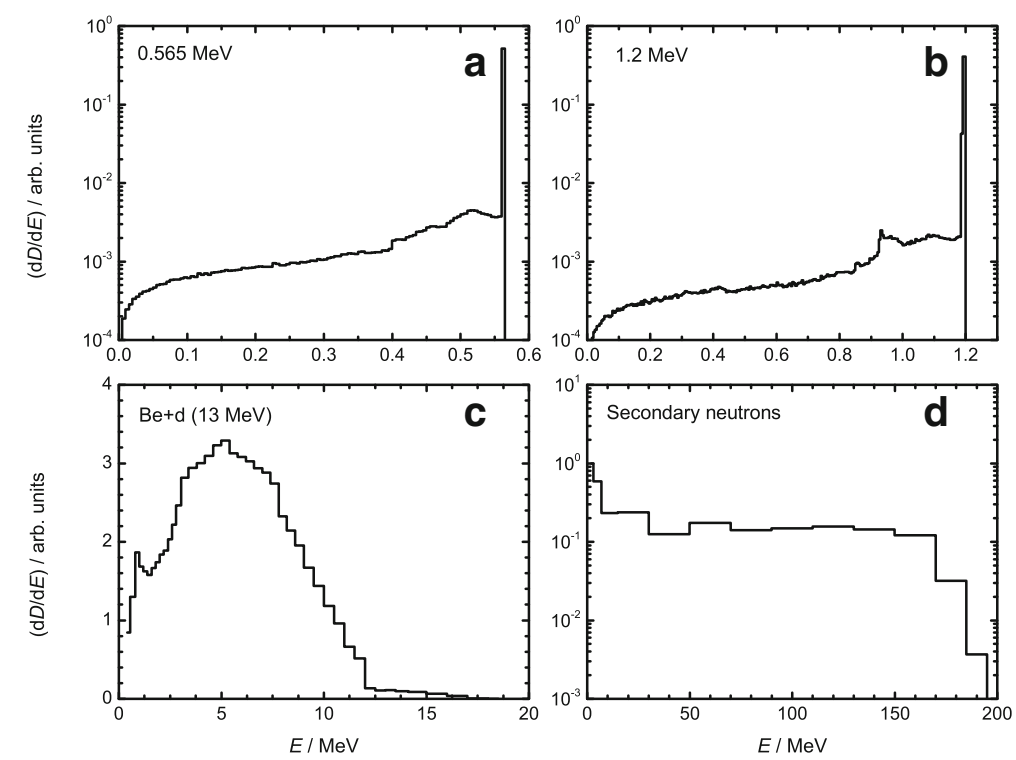

Fig. 1 Relative energy distributions of the four different neutron fields. a Low-energy monoenergetic neutrons of $0.56 \mathrm{MeV}$; b Low-energy monoenergetic neutrons of $1.2 \mathrm{MeV}$; c Medium-energy neutrons with a broad energy distribution and a mean neutron energy of $\left\langle E_{n}>=\right.$ $5.8 \mathrm{MeV}$; d Mixed gamma - secondary neutron field with a mean neutron energy of $<E_{n}>=70.5 \mathrm{MeV}$, produced by a $190 \mathrm{MeV}$ proton beam impinging on a water phantom 
Table 1 Neutron contribution for $1.2 \mathrm{MeV}$ and $0.56 \mathrm{MeV}$ monoenergetic neutrons

\begin{tabular}{cllll}
\hline $1.2 \mathrm{MeV}$ & & & & \\
$d / \mathrm{cm}$ & $K_{\mathrm{sc}} / K_{\text {dir }}$ & $<E_{\text {dir }}>/ \mathrm{MeV}$ & $<E_{\mathrm{sc}}>/ \mathrm{MeV}$ & $<E_{\text {tot }}>/ \mathrm{MeV}$ \\
5.27 & 0.127 & 1.190 & 0.821 & 1.149 \\
7.27 & 0.226 & 1.195 & 0.842 & 1.130 \\
10.27 & 0.253 & 1.197 & 0.881 & 1.134 \\
$0.56 \mathrm{MeV}$ & & & & \\
$d / \mathrm{cm}$ & $K_{\mathrm{sc}} / K_{\text {dir }}$ & $<E_{\text {dir }}>/ \mathrm{MeV}$ & $<E_{\mathrm{sc}}>/ \mathrm{MeV}$ & $<E_{\text {tot }}>/ \mathrm{MeV}$ \\
5.27 & 0.174 & 0.562 & 0.386 & 0.536 \\
7.27 & 0.332 & 0.563 & 0.394 & 0.521 \\
10.27 & 0.378 & 0.564 & 0.411 & 0.522 \\
\hline
\end{tabular}

Relative contribution of scattered neutrons to the kerma $K$ and the kermaweighted mean energies $\langle E>$ of the scattered neutrons and direct for 1.2 MeV and $0.56 \mathrm{MeV}$ monoenergetic neutrons. The subscripts 'sc' and 'dir' denote scattered and direct, i.e. uncollided, neutrons, respectively. The distance of the centre of the volume containing the cell suspension from the neutron source is denoted by $d$

was calibrated using a scintillation detector to determine the number of protons as function of the accumulated charge from the ionisation chamber. The absolute uncertainty in the number of protons entering the water phantom is estimated to be of the order of $1 \%$. This uncertainty is mainly due to the uncertainty in the determination of the calibration factor converting the accumulated charge from the ionization chamber to the number of protons entering the water phantom. Samples were positioned behind the water phantom (at $0^{\circ}$ relative to the incident proton beam) at a distance of $50 \mathrm{~mm}$. Proton interactions in water generated a mixed gamma - secondary neutron field at the sample positions. The total dose on the sample delivered by the mixed field was determined using a Monte Carlo simulation described below to be $4.0 \mathrm{E}-15 \mathrm{~Gy} /$ proton. Four sets of samples were irradiated with respectively $3.80 \mathrm{E} 13$; 9.50E13; $1.90 \mathrm{E} 14$ and $3.80 \mathrm{E} 14$ protons entering the water phantom, with total doses of 0.152, $0.38,0.76$ and 1.52 Gy, respectively. The dose rate was chosen such that each irradiation had equal duration $(5.5 \mathrm{~h})$, and that such duration was comparable to that for LDR irradiations at $\mathrm{PTB}$, in view of the final data comparison. The relative standard uncertainty for the total dose determination was about $5-6 \%$.

All radiation fields and sample exposures were simulated using the Monte Carlo radiation-transport code PHITS ver. 2.88 [24], verifying dose homogeneity in the containers, dose-distance relationships and characteristics of the neutron/photon field at the container location. For the irradiation setup at KVI-CART, the primary proton beam source of energy $190 \mathrm{MeV}$ was modelled as a Gaussian distribution in $x-y$ plane with full width at half maximum (FWHM) of $0.9 \mathrm{~cm}$. The energy spectrum (Fig. 1) of the secondary neutron field produced by a $190 \mathrm{MeV}$ proton beam impinging on a water phantom was simulated exactly at the cell position. The dose-averaged mean neutron energy at the cell position was calculated as $<E_{\mathrm{n}}>=70.5 \mathrm{MeV}$. The ratio of neutron dose/total dose was 0.65 , meaning $35 \%$ extra dose to the samples from gammas. This estimation of the neutron absorbed dose is done by tracking the recoil particles directly, and running PHITS in the mode that scores the energy loss of charged particles and nuclei. For neutron induced reactions below $20 \mathrm{MeV}$, PHITS was run in the Event Generator Mode using the Evaluated Nuclear Data libraries JENDL-4.0. [25]. For higher energy neutrons (and for other hadrons), the intra-nuclear cascade model INCL4.6 [26] was employed for simulating the dynamic stage of hadroninduced nuclear reactions. The quantum molecular dynamics model JQMD [27] was employed for nucleusinduced reactions. The evaporation and fission model GEM [28] was adopted for simulating the static stage for both hadron- and nucleus-induced reactions.

\section{Colony forming assay}

Twenty-four hours after IR, $1 \times 10^{3}$ cells were seeded in a $25 \mathrm{~cm}^{2}$ cell culture flask in triplicates for each dose value. Eight days later the colonies were fixed with $70 \%$ ethanol for $10 \mathrm{~min}$ and stained for 5-10 min with $1 \%$ crystal violet solution (Serva Electrophoresis $\mathrm{GmbH}$, Heidelberg, Germany). Colonies consisting of 50 cells and more were counted. Plating efficiency and survival fractions (SF) were determined and RBE values for a survival of $10 \%$, referred to as $\mathrm{RBE}_{(\mathrm{SF} \text { 0.1) }}$ in the text, were calculated with respect to X-rays (LDR) as described by Paganetti [29].

\section{Immunostaining of DSBs via $\mathrm{\gamma H} 2 \mathrm{AX}$ antibody}

Directly after irradiation, $1 \times 10^{4}$ cells per well $\left(1.8 \mathrm{~cm}^{2}\right)$ were seeded in duplicate in chamber slides (LabTek ${ }^{\circ}$, Nunc, Roskilde, Denmark) and incubated for $24 \mathrm{~h}$. After fixation with $2 \%$ formaldehyde and permeabilisation with 0.25\% triton-X 100 (both Sigma Aldrich Chemie GmbH, Munich, Germany) the cells were consecutively incubated 60 min with anti- $\gamma \mathrm{H} 2 \mathrm{AX}$ antibody (1:500, clone JBW301, Merck Millipore) and Alexa Fluor 594 goat anti-mouse IgG1 (1:400, Molecular Probes/Life Technologies, Darmstadt, Germany) for $30 \mathrm{~min}$. The slides were mounted with Vectashield ${ }^{\circ}$ containing anti-4',6-diamidino-2-phenylindole (DAPI; Vector Laboratories, Inc., Burlingame, CA). The foci were visualised with an Eclipse TE300 inverted microscope (Nikon, Tokyo, Japan). At a magnification of $1000 \times$, the foci of 50 cells per chamber were counted; two chambers per irradiation. The extra yield $(\Delta \mathrm{Y})$ was calculated as the difference between irradiated samples and the individual $0 \mathrm{~Gy}$ control value of residual foci as a function of dose and plotted in a graph. Linearisation was performed as described by Barendsen [30]. RBE values, 
referred to as $\mathrm{RBE}_{\text {(foci } 24 \mathrm{~h} \text { ) }}$ in the text, were calculated with respect to LDR X-rays via the $\alpha$ value with reference to Franken et al. [31, 32]. Fits to the data points using the eq. $F(D)=\alpha D+\beta D^{2}$ yielded $\beta$ values of zero.

\section{Sphere formation assay}

Twenty four hour after IR $1 \times 10^{4}$ cells per well were plated in triplicates in ultra-low attachment 6-well plates (Corning ${ }^{\ominus}$ Costar $^{\circ}$, Corning Incorporated, VWR, Darmstadt, Germany) for each irradiation dose. Since the cells can not adhere to the cell culture surface, they are able to form three-dimensional spheres. All samples were incubated under standard cell culture conditions. The number of spheres was counted by microscopy with a magnification of $100 \times$ at day $1-7$ after seeding, which is day $2-8$ after IR. The sham irradiated control ( 0 Gy) of each radiation quality was set to $100 \%$ at every counting day. The radiation-induced change in the number of the spheres was related to the appropriate 0 Gy control (100\%).

\section{Statistical analysis}

Data of at least three independent experiments are represented as mean values \pm standard error of the mean (SEM). For the clonogenic survival and for DNA DSBs, the assessment of statistical significance of differences was performed by student t-test. The statistical analyses of all values refer to LDR X-rays. The survival-/focivalues obtained from fits to the data points were used for statistical analyses between 0 and 2 Gy. A value of $p<0.05$ was considered to indicate a statistically significant difference. $\mathrm{F}$ spheres formation, the statistical significance to the individual sham-irradiated control (0 Gy) of each radiation quality was calculated via onesample $\mathrm{t}$-test and a value of $p<0.02$ indicated a statistically significant difference.

\section{Results}

Clonogenic survival after IR

Long-term effects after radiation were investigated via the clonogenic survival assay. For all radiation qualities a dose-dependent decrease in the SF (Fig. 2) was observed. Additional measurements up to 6 Gy were carried out for $\mathrm{X}$-rays in order to verify the linear-quadratic relation between dose and cell survival (Fig. 3). The SF after HDR Xray irradiation was about $67 \%$ after $1 \mathrm{~Gy}$ and $58 \%$ after 2 Gy; 1 Gy of X-rays LDR resulted only in a small decrease of SF (78\%). The strongest effects on the SF were observed after irradiations with low-energy monoenergetic neutrons of $0.56 \mathrm{MeV}$ and $1.2 \mathrm{MeV}$. There were significant changes for both monoenergetic neutron energies at $0.40 \mathrm{~Gy}$ (for $0.56 \mathrm{MeV}$ neutrons) and $0.42 \mathrm{~Gy}$ (for $1.2 \mathrm{MeV}$ neutrons), respectively, as well as at $0.84 \mathrm{~Gy}$ and $0.85 \mathrm{~Gy}(0.56 \mathrm{MeV}$; $1.2 \mathrm{MeV}$ ) compared to the $1 \mathrm{~Gy}$ of LDR X-rays (SF of $78 \%)$. The effect of $\mathrm{HDR}<E_{\mathrm{n}}>=5.8 \mathrm{MeV}$ neutrons was

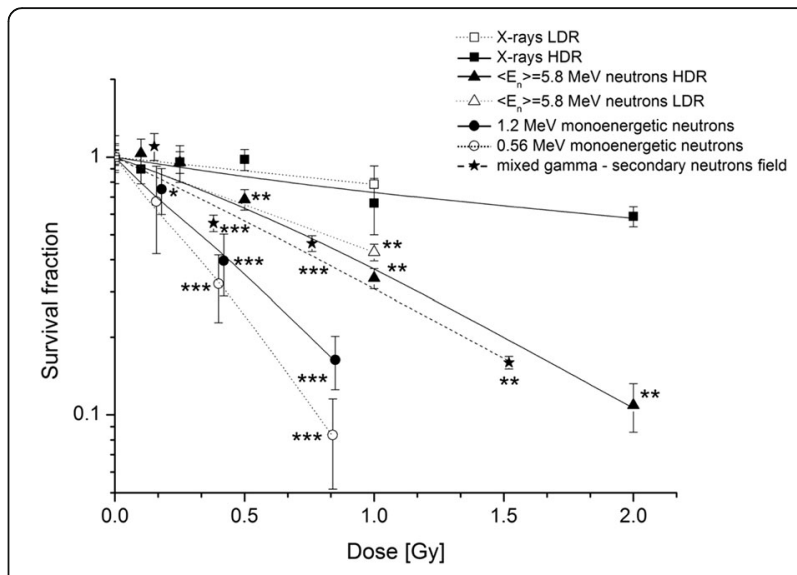

Fig. 2 Clonogenic survival of MCF10A cells after irradiation. Data from three (LDR $<E_{\mathrm{n}}>=5.8 \mathrm{MeV}$ neutrons, HDR and LDR X-rays, mixed gamma - secondary neutron $\left\langle E_{\mathrm{n}}>=70.5 \mathrm{MeV}\right.$ field), four ( $\mathrm{HDR}<E_{\mathrm{n}}>=$ $5.8 \mathrm{MeV}$ neutrons, $0.56 \mathrm{MeV}$ monoenergetic neutrons), and five (1.2 MeV monoenergetic neutrons) independent experiments, are presented as mean values \pm SEM of the survival fraction. The significances refer to the equal doses of $X$-rays LDR irradiation. Asterisks illustrate significances: ${ }^{*} p<0.05,{ }^{* *} p<0.01$, ${ }^{* * *} p<0.001$. (HDR, high dose rate; LDR, low dose rate)

slightly less pronounced: after a dose of 1 Gy the SF was $35 \%$. This effect was still significant compared to the survival after 1 Gy of LDR X-rays. With respect to 2 Gy of HDR X-rays, the SF was significantly decreased by a factor of 5 after 2 Gy of HDR medium-energy neutrons $\left(<E_{\mathrm{n}}>=5.8 \mathrm{MeV}\right)$. The effectiveness of $1 \mathrm{~Gy}$ of LDR medium-energy neutrons was higher compared to X-ray LDR as the SF was only $42 \%$. The mixed gamma - secondary neutrons had a comparable effect on the cells as HDR and LDR medium-energy neutrons $\left(<E_{\mathrm{n}}>=5.8 \mathrm{MeV}\right)$. After an IR of 1.52 Gy the SF was reduced to $20 \%$. RBE

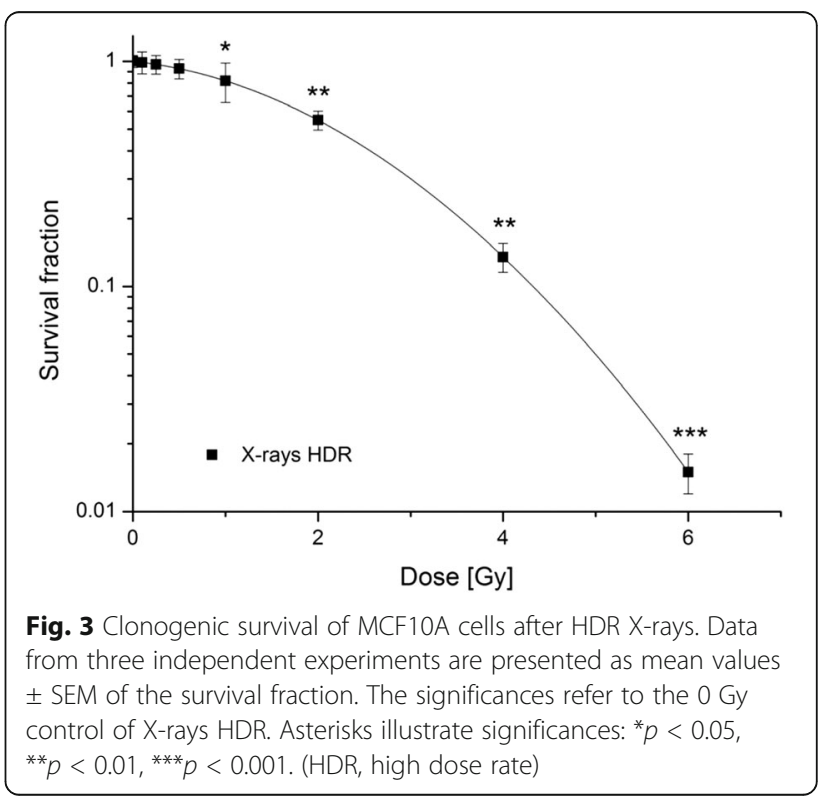


values were calculated using $220 \mathrm{kV}$ X-rays LDR as a reference (Table 2). Monoenergetic neutrons of $0.56 \mathrm{MeV}$ and $1.2 \mathrm{MeV}$ had the highest RBE values of 4.97 and 3.75 respectively. The RBE value of 2.09 for the mixed gamma - secondary neutrons $\left(\left\langle E_{\mathrm{n}}\right\rangle=70.5 \mathrm{MeV}\right)$ is similar to RBE values of 2.06 and 1.99 for HDR and LDR mediumenergy neutrons $\left(<E_{\mathrm{n}}>=5.8 \mathrm{MeV}\right)$.

\section{Residual $\mathrm{\gamma H} 2 \mathrm{AX}$ foci induction by IR}

Radiation-induced residual $\gamma \mathrm{H} 2 \mathrm{AX}$ foci, used as markers for complex DSBs, were detected $24 \mathrm{~h}$ following irradiations, when repair of less complex lesions is supposed to be completed and a higher complexity of residual lesions can be assumed. The extra yield of foci was plotted with respect to sham conditions of each radiation type. The number of foci increased with higher doses for all radiation qualities (Fig. 4). Increasing doses (HDR and LDR) of $220 \mathrm{kV}$ X-rays caused low but significant increases in the mean number of DSBs within the cell nuclei. Monoenergetic neutrons of $1.2 \mathrm{MeV}$ as well as HDR and LDR medium-energy neutrons produced much more damage in terms of DSBs as LDR and HDR X-rays. The exposure to a mixed gamma - neutron field with secondary neutrons of $\left\langle E_{\mathrm{n}}\right\rangle=70.5 \mathrm{MeV}$, as performed at KVI-CART, induced an almost similar effect as HDR neutrons of $<E_{\mathrm{n}}>=5.8 \mathrm{MeV}$. The $\alpha$-based RBE values for foci induction showed a very clear increase following neutron radiation exposure with HDR and LDR medium-energy neutrons, low-energy neutrons of $1.2 \mathrm{MeV}$ and the mixed gamma - secondary neutron field produced by a $190 \mathrm{MeV}$ proton beam, when compared to X-rays. The values were even higher when the cells were irradiated with monoenergetic neutrons of $0.56 \mathrm{MeV}$ (Table 2).

\section{Formation of spheres after IR}

The influence of IR on the sphere formation ability of human mammary epithelial cells was examined using the sphere formation assay. This assay was performed $24 \mathrm{~h}$ after irradiation (Fig. 5). For low-energy neutrons and for the mixed gamma - secondary neutron field the values for the highest radiation dose (0.88 Gy for $1.2 \mathrm{MeV}$ neutrons,
$0.82 \mathrm{~Gy}$ for $0.56 \mathrm{MeV}$ neutrons, $1.52 \mathrm{~Gy}$ for the mixed field) and for medium-energy neutrons and X-rays the values for 1 Gy were normalized to the 0 Gy control, which was set to $100 \%$ for each day (see 100\% baseline in the graph).

With respect to the individual 0 Gy control per day, there is a general decrease of the sphere formation ability visible for every radiation quality. The irradiation with LDR X-rays showed a clear reduction of the sphere formation ability at day 2 and 4, unlike HDR-X-rays, where no significant impairments could be observed. The exposure to neutrons caused significant changes. The use of HDR neutrons $\left(<E_{\mathrm{n}}>=5.8 \mathrm{MeV}\right)$ showed a slightly stronger effect, especially on day 4 , as the LDR neutrons, which showed a uniform reduction at all days, which is in the range of $63-73 \%$. Radiation with 0.88 Gy of $1.2 \mathrm{MeV}$ monoenergetic neutrons and $1.52 \mathrm{~Gy}$ of a mixed gamma-secondary neutron field showed timedependent a reducing effect on the sphere formation ability. On the eighth day, the ability to form spheres is more restricted than on the second day after irradiation. In addition, the exposure to $0.56 \mathrm{MeV}$ monoenergetic neutrons resulted in a strong reduction of the sphere formation ability already 2 days after irradiation but this decrease seemed to recover within the following 6 days.

\section{Discussion}

In order to investigate the RBE of neutrons relative to photons as a function of neutron energy, the present study examined the different radiobiological effects following neutron and X-ray radiation. We also measured the RBE of a mixed gamma - secondary neutron field, generated by interactions of $190 \mathrm{MeV}$ protons in water, with a neutron dose equal to $65 \%$ of the total dose, and neutrons with an energy spectrum similar to that produced in a clinical setting for proton therapy. Currently, there are limited systematic studies on the effects of neutron exposure as a function of neutron energy on normal tissue cells, highlighting the comparison of neutrons to photons (an example in this sense is the study by Göhde et al. with neutron energies of $0.56 \mathrm{MeV}$, 2.5 $\mathrm{MeV}$ and 14.8 $\mathrm{MeV}$ [33]).

Table 2 Values of the a-component of LQ model for SF and DSBs for different radiation qualities

\begin{tabular}{|c|c|c|c|c|}
\hline & $\begin{array}{l}\text { a values } \\
\text { of SF }\end{array}$ & $\begin{array}{l}\text { a values } \\
\text { of foci } 24 \mathrm{~h}\end{array}$ & $\left.\mathrm{RBE}_{(\mathrm{SF}} 0.1\right)$ & $\operatorname{RBE}_{\text {(foci } 24 h \text { ) }}$ \\
\hline 220 kV X-rays LDR, reference & 0.12 & 0.84 & 1.00 & 1.00 \\
\hline 220 kV X-rays HDR & 0.24 & 1.87 & 0.58 & 2.22 \\
\hline$<E_{\mathrm{n}}>=5.8 \mathrm{MeV}$ neutrons LDR & 0.43 & 4.43 & 2.06 & 5.25 \\
\hline$<E_{\mathrm{n}}>=5.8 \mathrm{MeV}$ neutrons HDR & 0.65 & 3.86 & 1.99 & 4.57 \\
\hline 1.2 MeV monoenergetic neutrons & 1.91 & 3.36 & 3.75 & 3.98 \\
\hline $0.56 \mathrm{MeV}$ monoenergetic neutrons & 2.55 & 6.71 & 4.97 & 7.95 \\
\hline mixed gamma - secondary neutron $\left(<E_{n}>=70.5 \mathrm{MeV}\right)$ field & 1.03 & 3.77 & 2.09 & 4.47 \\
\hline
\end{tabular}

$\mathrm{RBE}_{(S F}$.1) are calculated for a survival fraction of $10 \%, \mathrm{RBE}_{(\text {(foci } 24 \mathrm{~h} \text { ) }}$ are $a$-based calculated according to $F(D)=a D+\beta D^{2}$, with $\beta$ equal to zero [31, 32] 


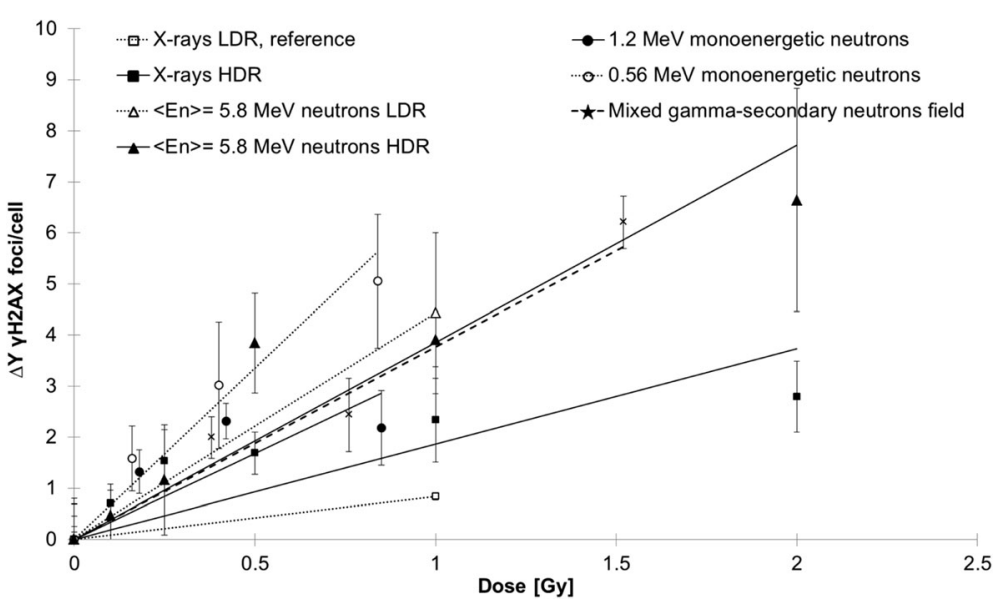

Fig. $4 \mathrm{DNA}$ double-strand breaks $24 \mathrm{~h}$ after irradiation. Extra yield $\triangle \mathrm{Y}$ (difference over the individual $0 \mathrm{~Gy}$ control value) of residual $\gamma H 2 \mathrm{AX}$ foci as a function of dose scored in MCF10A cells $24 \mathrm{~h}$ after radiation. Data from three (mixed gamma - secondary neutron $\left\langle E_{\mathrm{n}}\right\rangle=70.5 \mathrm{MeV}$ field and $X$-rays) and four (medium-energy $\left\langle E_{n}>=5.8 \mathrm{MeV}\right.$ neutrons, $1.2 \mathrm{MeV}$ and $0.56 \mathrm{MeV}$ monoenergetic neutrons) independent experiments are presented as mean values $\pm S E M$. Fitted with $L Q$ model $F(D)=a D+\beta D^{2}$, with $\beta$ equal to zero [30-32]. The significances refer to the equal doses of X-rays LDR irradiation. Asterisks illustrate significances: ${ }^{*} p<0.05,{ }^{* * *} p<0.001$. (HDR, high dose rate; LDR, low dose rate)

Concerning clonogenic survival, our data showed a large variation between the effects of various neutron energies with regard to the use of doses up to 1 Gy. Presented results about the radiobiological effects on the clonogenic survival with low-energy monoenergetic neutrons are of particular significance in contrast to X-rays and medium-energy neutrons $\left(<E_{\mathrm{n}}>=5.8 \mathrm{MeV}\right)$. Compared to X-rays (LDR), the SF was significantly decreased after irradiation with monoenergetic neutrons $(0.56 \mathrm{MeV}, 1.2 \mathrm{MeV})$. This effectiveness of $0.56 \mathrm{MeV}$ neutrons on the clonogenic survival confirms the results of Okumura et al. [34]. Frankenberg-Schwager et al. [22] obtained an RBE of 5.4 for a different cell line, experimental conditions and reference radiation quality. These studies confirm and support our results on the effect of neutrons, but lack the representation of human tissue specific cells, using non-human or hybrid cell lines and also a smaller range of applied doses. The present study quantifies the long-term effects of neutrons with different energies and doses on normal human tissue cells from the mammary gland.

A common feature of our study and others $[22,34]$ is the use of cell suspensions for the irradiation. A study investigating different cell culture models used during the irradiation was led by Cansolino and colleagues [35]. Here, the SF for cell suspension and adherent cells was determined for rat colon adenocarcinoma cell lines. In accordance to our data, they showed a higher impact of neutrons (up to $10 \mathrm{~Gy}$ ) on suspended cells compared to ${ }^{60} \mathrm{Co}$.

Furthermore, next to energy, radiation type and doses, the dose rate can play an important role for the radiobiological effects, especially regarding the induction of residual

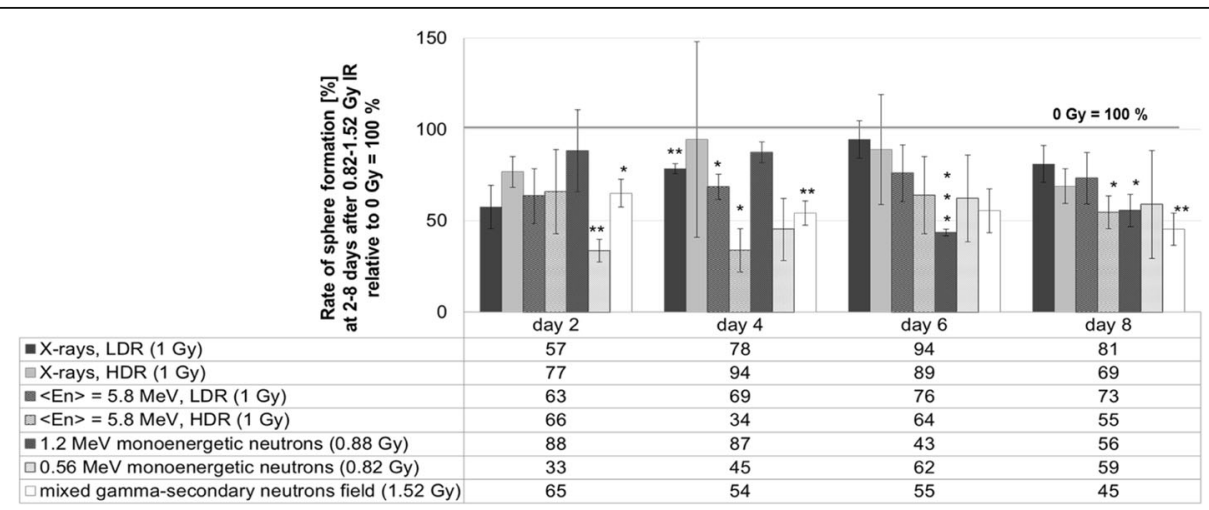

Fig. 5 Sphere formation after irradiation. Change of the sphere formation ability of MCF10A cells at different time points after exposure to X-rays and neutrons with respect to individual 0 Gy control per day ( $=100 \%$ baseline). Data from three independent experiments are presented as mean values \pm SEM. Significances refer to the individual 0 Gy control of each radiation quality per day. Asterisks illustrate significances: ${ }^{*} p<0.02$, ${ }^{* *} p<0.01,{ }^{* * *} p<0.002$. (HDR, high dose rate; LDR, low dose rate) 
$\gamma \mathrm{H} 2 \mathrm{AX}$ foci. For DNA damage, represented by $\gamma \mathrm{H} 2 \mathrm{AX}$ foci (DSBs) $24 \mathrm{~h}$ after radiation exposure, our findings reveal a 2-fold higher response of cells irradiated with HDR in contrast to LDR X-rays. This can be a result of repair processes, which start after a few minutes. Therefore, DSBs induced by LDR may already be partially repaired during the irradiation process before the full applicable dose is reached, as the time for a dose of 1 Gy generated with LDR is more than 20-times longer than generated with HDR [36].

Our results demonstrated that the number of residual $\gamma \mathrm{H} 2 \mathrm{AX}$ foci $24 \mathrm{~h}$ after IR, as an indicator for DSBs, increased as a function of increasing dose, which has been also reported by Okumura and colleagues [34], who observed 53BP1 foci as an indicator for DSBs for several time points. In contrast, they could not identify a difference between samples (1-3 h following IR) exposed to neutrons (mixed beam) and $\gamma$-rays, however, they did not examine the residual $\gamma \mathrm{H} 2 \mathrm{AX}$ foci $24 \mathrm{~h}$ after radiation. Just as Tanaka et al. observed DNA damage by means of comet assay [37], we could also demonstrate a higher biological effectiveness of $0.56 \mathrm{MeV}$ monoenergetic neutrons compared to $1.2 \mathrm{MeV}$ neutrons. Their analysis of DNA damage demonstrated the same pattern of effectiveness of the different radiation qualities.

Using a sphere formation assay, we examined the radiation-induced response of a potential stem-like subpopulation: it is known that MCF10A cells include a progenitor like cell subpopulation [15]. Present data showed alterations in the sphere formation ability, which were more evident following monoenergetic neutron irradiation and following the exposure to the mixed gamma - secondary neutron field, compared to X-rays and medium-energy neutrons $\left(<E_{\mathrm{n}}>=5.8 \mathrm{MeV}\right)$. Like Dionet et al., who investigated fast neutrons on normal skin fibroblast by cell survival assay, we could show a stronger effect following HDR neutrons compared to LDR neutrons [38]. In general, most publications concerning sphere formation combining mammary cells and radiation are dealing with cancer cell lines [39, 40]. The present study gives instead a first insight on the sphere formation ability of normal human breast cells irradiated with a broad range of neutron energies.

From the presented dataset, observable differences in $\mathrm{RBE}$ values by various neutron energies relative to $\mathrm{X}$ rays can be concluded.

Consistent with Tanaka et al. [37] and Schmid et al. [23], we obtained results with increasing RBE values as a function of decreasing neutron energies (between $0.56 \mathrm{MeV}$ and $\left\langle E_{\mathrm{n}}>=5.8 \mathrm{MeV}\right)$. The mixed gamma - secondary neutron $\left(<E_{\mathrm{n}}>=70.5 \mathrm{MeV}\right)$ field, generated by protons of $190 \mathrm{MeV}$ impinging on a water phantom, yielded RBE values for both, SF and residual foci, which are comparable to those of HDR and LDR medium-energy neutrons of $\left\langle E_{\mathrm{n}}\right\rangle=5.8 \mathrm{MeV}$ : 4.47 for $\mathrm{RBE}_{(\text {foci } 24 \mathrm{~h})}$ and 2.09 for $\mathrm{RBE}_{(\mathrm{SF}}$ 0.1). As well known, RBE is a variable function of several factors, among which the endpoint itself [37, 41]. Qualitative consistency is found in this study between $\mathrm{RBE}_{(\mathrm{SF} \text { 0.1) }}$ and $\mathrm{RBE}_{\text {(foci } 24 \mathrm{~h} \text { ), }}$ both increasing with decreasing neutron energies for the covered range from $0.56 \mathrm{MeV}$ to $<E_{\mathrm{n}}>=5.8 \mathrm{MeV}$.

\section{Conclusions}

The present study extensively investigated chosen radiobiological effects following exposures in the dose range of 0 Gy up to 2 Gy to different neutron energies compared to X-rays in MCF10A normal human breast cells. The range of selected neutron energies $(0.56 \mathrm{MeV}, 1.2 \mathrm{MeV}$ and a broad spectrum with a mean energy of $5.8 \mathrm{MeV}$ ) was expanded by the use of a mixed gamma - secondary neutron field $\left.\left(<E_{\mathrm{n}}\right\rangle=70.5 \mathrm{MeV}\right)$, adopted to simulate the scattered neutron field during proton therapy. Dose-rate effects were also addressed when high vs. low dose rate exposures could be performed (X-rays and mediumenergy neutron exposure). Effects on clonogenic survival and $\gamma \mathrm{H} 2 \mathrm{AX}$ residual foci induction are reported, which were strongly dependent on radiation quality and dose (dose rate for residual foci induction only). RBE values were extracted from measured endpoints as $\mathrm{RBE}_{(\mathrm{SF}}$ 0.1)

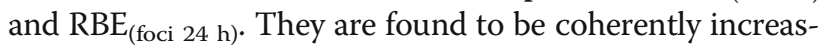
ing for decreasing neutron energy in the investigated energy range. The exposure to the mixed gamma secondary neutron field yield RBEs as high as for medium-energy neutrons. The response of the potential fraction of stem-like cells in the MCF10A cell population was also addressed, by measuring sphere formation ability for up to 8 days after exposure with the maximal dose or 1 Gy for each radiation quality ( $0.88 \mathrm{~Gy}-1.52 \mathrm{~Gy})$.This investigation provides a deeper insight into the radiobiological effects of neutron exposure, which is very important in order to assess the risk of secondary neutrons produced during conventional and particle radiotherapy and their possible trigger function for potential carcinogenic effects on normal breast cells and stem cells.

\section{Additional file}

Additional file 1: Authentification certification of MCF10A cell line. (PDF $1337 \mathrm{~kb})$

\section{Abbreviations}

BSA: Bovine serum albumin; $\mathrm{CO}_{2}$ : Carbon dioxide; DAPI: 4',6-diamidino-2phenylindole; DMEM: Dulbecco's modified Eagle medium; DSB: Double strand break; EGF: Epidermal growth factor; HDR: High dose rate; IR: Ionising radiation; LDR: Low dose rate; PBS: Phosphate buffered saline;

PMMA: Polymethylmethacrylate; RBE: Relative biological effectiveness; SF: Survival fraction; $\mathrm{YH} 2 \mathrm{AX}$ : Phosphorylated histone $2 \mathrm{AX}$

\section{Acknowledgements}

The authors would like to thank the operators and technical staff of the PTB accelerator facility in Braunschweig and of the CART, Groningen, and members from ANDANTE for support and planning. We acknowledge financial support by Deutsche Forschungsgemeinschaft and Universität 
Rostock/Universitätsmedizin Rostock within the funding programme Open Access Publishing.

\section{Funding}

This work was financially supported by funding from European Community's Seventh Framework Programme (EURATOM) contract FP7-295970 (ANDANTE).

\section{Availability of data and materials}

The datasets used and/or analysed during the current study available from the corresponding author on reasonable request.

\section{Authors' contributions}

DJ and MZ performed all experiments, acquired and analysed the data, wrote and drafted the manuscript. UG, RN, MP, GB and MvG performed the dosimetry of neutron irradiations. UG and RN supervised the neutron irradiations at PTB. MvG supervised radiations at KVI-CART. SK performed the dosimetry for X-rays. MP and GB performed Monte Carlo simulations. UG, RN, $\mathrm{GB}$ and MP critically revised the manuscript. $\mathrm{KM}$ and $\mathrm{GH}$ designed the study. $\mathrm{GH}$ contributed to materials and tools. All authors read and approved the final manuscript.

\section{Ethics approval and consent to participate} Not applicable.

\section{Consent for publication}

Not applicable.

\section{Competing interests}

The authors declare that they have no competing interests.

\section{Publisher's Note}

Springer Nature remains neutral with regard to jurisdictional claims in published maps and institutional affiliations.

\section{Author details}

'Department of Radiotherapy and Radiation Oncology, University Medical Centre Rostock, Suedring 75, 18059 Rostock, Germany.

${ }^{2}$ Physikalisch-Technische Bundesanstalt (PTB), Bundesallee 100, 38116 Braunschweig, Germany. ${ }^{3}$ Physics Department, University of Pavia, Via Bassi 6, 27100 Pavia, Italy. ${ }^{4}$ Technische Universität Wien, Atominstitut, Stadionallee 2. 1020 Vienna, Austria. ${ }^{5} \mathrm{KVI}$ - Center for Advanced Radiation Technology (KVI-CART), Zernikelaan 25, 9747 AA Groningen, The Netherlands.

\section{Received: 15 March 2017 Accepted: 18 September 2017}

\section{Published online: 25 September 2017}

\section{References}

1. Newhauser WD, Durante M. Assessing the risk of second malignancies after modern radiotherapy. Nat Rev Cancer. 2011;11:438-48. Nature Publishing Group

2. Paganetti H, Athar BS, Moteabbed M, Adams AJ, Schneider U, Yock Tl. Assessment of radiation-induced second cancer risks in proton therapy and IMRT for organs inside the primary radiation field. Phys Med Biol. 2012;57:6047-61.

3. Chung CS, Yock TI, Nelson K, Xu Y, Keating NL, Tarbell NJ. Incidence of second malignancies among patients treated with proton versus photon radiation. Int J Radiat Oncol Biol Phys. Elsevier Inc. 2013;87:46-52.

4. Zhang R, Howell RM, Giebeler A, Taddei PJ, Mahajan A, Newhauser WD. Comparison of risk of radiogenic second cancer following photon and proton craniospinal irradiation for a pediatric medulloblastoma patient. Phys Med Biol. 2013:58:807-23.

5. Suit H, Goldberg S, Niemierko A, Ancukiewicz M, Hall E, Goitein M, et al. Secondary carcinogenesis in patients treated with radiation: a review of data on radiation-induced cancers in human, non-human primate, canine and rodent subjects. Radiat Res. 2007;167:12-42.

6. Bhatia S, Robison LL, Oberlin O, Greenberg M, Bunin G, Fossati-Bellani F, et al. Breast cancer and other second neoplasms after childhood Hodgkin's disease. N Engl J Med. 1996:334:745-51.

7. Greenfield DM, Wright J, Brown JE, Hancock BW, Davies H a, OToole L, et al. High incidence of late effects found in Hodgkin's lymphoma survivors, following recall for breast cancer screening. Br J Cancer. 2006;94:469-72.
8. Koo E, Henderson M, Dwyer M, Skandarajah AR. Management and Prevention of Breast Cancer After Radiation to the Chest for Childhood, Adolescent, and Young Adulthood Malignancy. Ann Surg Oncol. 2015; 22:545-51.

9. Schellong G, Riepenhausen M, Ehlert K, Brämswig J, Dörffel W, Schmutzler RK, et al. Brustkrebs bei jungen Frauen nach Therapie eines Hodgkinlymphoms im Kindes- und Jugendalter: Eine Beobachtungsstudie mit bis zu 33 Jahren Follow-Up. Dtsch Arztebl Int. 2014;111:3-9.

10. Brenner DJ, Hall EJ. Secondary neutrons in clinical proton radiotherapy: A charged issue. Radiother Oncol. 2008;86:165-70.

11. Loebrich M, Shibata A, Beucher A, Fisher A, Ensminger M, Goodarzi A a, et al. Gamma H2AX foci analysis for monitoring DNA double-strand break repair: Strengths, limitations and optimization. Cell Cycle. 2010;9:662-9.

12. Mariotti LG, Pirovano G, Savage Kl, Ghita M, Ottolenghi A, Prise KM, et al. Use of the gamma-H2AX assay to investigate DNA repair dynamics following multiple radiation exposures. PLoS One. 2013;8:1-12.

13. Rothkamm K, Horn S. Gamma-H2AX as protein biomarker for radiation exposure. Ann Ist Super Sanita. 2009;45:265-71.

14. Stingl J. Detection and analysis of mammary gland stem cells. J Pathol. 2009;217:229-41.

15. Tang J, Fernandez-Garcia I, Vijayakumar S, Martinez-Ruis H, Illa-Bochaca I, Nguyen $\mathrm{DH}$, et al. Irradiation of juvenile, but not adult, mammary gland increases stem cell self-renewal and estrogen receptor negative tumors. Stem Cells. 2014;32:649-61.

16. Dontu G, Abdallah WM, Foley JM, Jackson KW, Clarke MF, Kawamura MJ, et al. In vitro propagation and transcriptional profiling of human mammary stem/progenitor cells. Genes Dev. 2003;17:1253-70.

17. Soule HD, Maloney TM, Wolman SR, Peterson WDJ, Brenz R, McGrath CM, et al. Isolation and characterization of a spontaneously immortalized human breast epithelial cell line, MCF-10. Cancer Res. 1990:50:6075-86.

18. Brede HJ, Dietze G, Kudo K, Schrewe UJ, Tancu F, Wen C. Neutron yields from thick Be targets bombarded with deuterons or protons. Nucl Inst Methods Phys Res A. 1989;274:332-44.

19. ICRU. Nuclear Data for Neutron and Proton Radiotherapy and for Radiation Protection. ICRU Report 63. Bethesda: International Commission on Radiation Units and Measurements; 2000.

20. Seltzer SM, Bartlett DT, Burns DT, Dietze G, Menzel H-G, Paretzke HG, et al. Fundamental Quantities And Units For lonizing Radiation (Revised) ICRUreport No 85. J ICRU. 2011;11:1-35.

21. ICRU Clinical neutron dosimetry, part I: determination of absorbed dose in a patient treated by external beams of fast neutrons. ICRU Report 45. Bethesda: International Commission on Radiation Units and Measurements; 1989.

22. Frankenberg-Schwager M, Spieren S, Pralle E, Giesen U, Brede HJ, Thiemig $\mathrm{M}$, et al. The RBE of $3.4 \mathrm{MeV}$-particles and $0.565 \mathrm{MeV}$ neutrons relative to $60 \mathrm{Co}$-rays for neoplastic transformation of human hybrid cells and the impact of culture conditions. Radiat Prot Dosim. 2010;138:29-39.

23. Schmid E, Schlegel D, Guldbakke S, Kapsch RP, Regulla D. RBE of nearly monoenergetic neutrons at energies of $36 \mathrm{keV}-14.6 \mathrm{MeV}$ for induction of dicentrics in human lymphocytes. Radiat Environ Biophys. 2003;42:87-94.

24. Sato T, Niita K, Matsuda N, Hashimoto S, Iwamoto Y, Noda S, et al. Particle and Heavy Ion Transport code System, PHITS, version 2.52. J Nucl Sci Technol. 2013;529:913-23.

25. Shibata K, Iwamoto O, Nakagawa T, Iwamoto N, Ichihara A, Kunieda S, et al. JENDL-4.0: A New Library for Nuclear Science and Engineering. J Nucl Sci Technol. 2011;48:1-30.

26. Boudard A, Cugnon J, David JC, Leray S, Mancusi D. New potentialities of the Liège intranuclear cascade model for reactions induced by nucleons and light charged particles. Phys Rev C - Nucl Phys. 2013;87:014606.

27. Niita K, Chiba S, Maruyama T, Maruyama T, Takada H, Fukahori T, et al. Analysis of the $(N, x N)$ reactions by quantum molecular dynamics plus statistical decay model. Phys Rev C. 1995;52:2620-35.

28. Furihata S. Statistical analysis of light fragement production from medium energy proton-induced reactions. Nucl Instruments Methods Phys Res B. 2000;171:251-8

29. Paganetti $\mathrm{H}$. Relative biological effectiveness (RBE) values for proton beam therapy. Variations as a function of biological endpoint, dose, and linear energy transfer. Phys Med Biol. 2014;59:R419-72.

30. Barendsen GW. Dose fractionation, dose rate and iso-effect relationships for normal tissue responses. Int J Radiat Oncol Biol Phys. 1982:8:1981-97. 
31. Franken NA, ten Cate R, Krawczyk PM, Stap J, Haveman J, et al. Comparison of RBE values of high-LET a-particles for the induction of DNA-DSBS, chromosome aberrations and cell reproductive death. Radiat Oncol. BioMed Central Ltd. 2011;6:64.

32. Franken N, Ruurs P, Ludwików G, van Bree C, Kipp JB, Darroudi F, et al. Correlation between cell reproductive death and chromosome aberrations assessed by FISH for low and high doses of radiation and sensitization by iodo-deoxyuridine in human SW-1573 cells. Int J Radiat Biol. 1999;75:293-9.

33. Göhde W, Uthe D, Wedemeyer N, Severin E, Greif K, Schlegel D, et al. Mutagenic effect of low energy neutrons on human chromosome 11. Int J Radiat Biol. 2003;79:911-8.

34. Okumura K, Kinashi Y, Kubota Y, Kitajima E, Okayasu R, Ono K, et al. Relative biological effects of neutron mixed-beam irradiation for boron neutron capture therapy on cell survival and DNA double-strand breaks in cultured mammalian cells. J Radiat Res. 2013;54:70-5.

35. Cansolino L, Clerici AM, Zonta C, Dionigi P, Mazzini G, Di Liberto R, et al. Comparative study of the radiobiological effects induced on adherent vs suspended cells by BNCT, neutrons and gamma rays treatments. Appl Radiat Isot. 2015;106:226-32.

36. Ishizaki K, Hayashi Y, Nakamura H, Yasui Y, Komatsu K, Tachibana A. No induction of p53 phosphorylation and few focus formation of phosphorylated H2AX suggest efficient repair of DNA damage during chronic low-dose-rate irradiation in human cells. J Radiat Res. 2004;45:521-5.

37. Tanaka K, Gajendiran N, Endo S, Komatsu K, Hoshi M, Kamada N. Neutron energy-dependent initial DNA damage and chromosomal exchange. J Radiat Res. 1999:40(Suppl):36-44.

38. Dionet C, Muller-Barthelemy M, Marceau G, Denis J-M, Averbeck D, Gueulette J, et al. Different dose rate-dependent responses of human melanoma cells and fibroblasts to low dose fast neutrons. Int J Radiat Biol. 2016;92:527-35.

39. Chang H-W, Wang H-C, Chen C-Y, Hung T-W, Hou M-F, Yuan S-S, et al. 5Azacytidine Induces Anoikis, Inhibits Mammosphere Formation and Reduces Metalloproteinase 9 Activity in MCF-7 Human Breast Cancer Cells. Molecules. 2014;19:3149-59.

40. Phillips TM, McBride WH, Pajonk F. The response of CD24-/low/CD44+ breast cancer-initiating cells to radiation. J Natl Cancer Inst. 2006:98:1777-85.

41. Durante M. New challenges in high-energy particle radiobiology. Br J Radiol. 2014:87:20130626

\section{Submit your next manuscript to BioMed Central and we will help you at every step:}

- We accept pre-submission inquiries

- Our selector tool helps you to find the most relevant journal

- We provide round the clock customer support

- Convenient online submission

- Thorough peer review

- Inclusion in PubMed and all major indexing services

- Maximum visibility for your research

Submit your manuscript at www.biomedcentral.com/submit

) Biomed Central 